\title{
THE TECHNOLOGY OF IMPROVING FLUID SPEECH AND MUSTERING THE STUTTER SPEAKING CHILDERN
}

\author{
Makhamova Umida Abdusattarovna ${ }^{1}$ \\ ${ }^{I}$ Toshkent State Pedagogical University independent researcher, speech therapist
}

\begin{abstract}
The article identifies the process of speech therapy for stuttering children's speech: the causes of stuttering, collection of anamnesis of children with stuttering speech defects, comprehensive examination of various aspects of speech, identification of the mechanism of developmental disorders and levels of stuttering using speech therapy-corrective technologies. Through this examination, the problems of speech defects in children in a timely manner were considered, and the purpose of the article was to reveal the concepts of stuttering, their comprehensive examination and methods of overcoming stuttering.

Keywords: speech, child, speech defects, stuttering, anamnesis, examination, upbringing, speech therapistcorrection-development, complex, degree, high, medium, low, decisions, methodologies, training, medical, psychological, technology, result.
\end{abstract}

\section{INTRODUCTION}

Decree of the President of the Republic of Uzbekistan Sh.M.Mirziyoev PF 4947 "On the Strategy for further development of the Republic of Uzbekistan" for 2017-2021, December 29, 2016 "On measures to further improve the system of preschool education in 2017-2021" Resolution No. PQ-2707 of September 9, 2017 "On measures to radically improve the system of preschool education" PQ-3261, "On measures to further develop the activities of non-governmental educational services" PQ-3276, "Preschool education We hope that this article will contribute to the further improvement of this system and the implementation of the tasks set out in other relevant regulations. Issues of upbringing and education of children with disabilities play an important role in the education system. Thanks to the organization of correctional education, treatment and social adaptation and rehabilitation, such individuals become active participants in socially useful work, as a result of which they are not neglected. The expansion and stratification of the system of special institutions for children with developmental disabilities in Uzbekistan necessitates the provision of special schools with highly qualified teachers-defectologists. In the system of preschool education, the network of special preschools is gradually expanding, a number of scientific and pedagogical institutions for the social protection of children with disabilities and developmental delays have been created and are being created.

Proper development of speech is of great importance for the active participation of every child in preschool education as a well-rounded person in accordance with the requirements of the times. Because speech is a tool of interpersonal communication, national wealth and education is a powerful tool for education and creativity. Therefore, everyone should be taught to master the speech, to fully express their opinion. The child learns to pronounce sounds and words correctly by imitating adults. This means that parents and educators need to pay attention to the correct pronunciation of sounds in children and in time to prevent the shortcomings and deficiencies that they encounter in speech.

In general, most preschoolers and school-age children will have clear and accurate speech. But despite this, there are also children with speech defects among them. It is children who stutter from those who have such a speech impediment.

Stuttering is a disorder of the speed of speech as a result of contraction of the muscles of the speech apparatus.

The problem of stuttering can be considered one of the oldest in the history of the development of the doctrine of speech disorders. Different interpretations of its essence are related to the development of science and the extent to which the authors have approached and are approaching such speech disorders. Much work has been done to eliminate stuttering in Russian children of preschool age. (G.A.Volkova, L.P.Uspenskaya, E.F.Rau, N.A.Chevelyova, V.I.Selevorstov, S.A.Mironova and others).

For the first time in Uzbekistan on the topic "Elimination of stuttering in schools and adults" Muminova L.R. defended his Ph.D. dissertation. The work on the elimination of stuttering in Uzbek children "Elimination of stuttering in preschool children through play" Pulatova S. defended his master's dissertation for the first time.

To date, the elimination of special speech deficiencies in kindergartens, the number of stuttering children of preschool age is increasing year by year, and the absence of methodological abiodies, ie programs, developments, methodological recommendations and manuals in groups opened for special stuttering children proves the relevance and lack of this topic. 


\section{MATERIALS AND METHODS}

Examination of the smoker is carried out in a comprehensive manner (speech therapist, neuropathologist, psychologist) with the involvement of other specialists, pediatricians, otolaryngologists and others, as needed.

The content of the examination is anamnestic data. The study of pedagogical psychological and medical documentation also includes self-examination of the stutterer.

In conversations with parents, we identified the most important events that took place in the family and, in this regard, identified the general, motor and speech development characteristics of the child.

The main aspects of the period of childbirth: the age of the mother at birth ( 35 years or older), mental health, illness of the parent, the course of pregnancy is assessed. Information about the health of the father and mother before the birth of the child allows to identify deviations in his physical and mental state. Identifying adverse factors in the development of the child in the mother's womb will help to determine their negative impact on the subsequent development of the child's speech.

The purpose of the examination is to gain a full understanding of the child's psycho-physical development and speech capabilities. It consists of anamnestic data and of course a speech check.

The speech therapist will work on the following points:

1. parental health.

2. the general development of the child before the onset of stuttering.

3. speech development.

4. features of raising a child in the family.

5. speech of others.

6. when the stuttering occurred.

7. what other speech defects were there during this period.

8. The features at which stuttering occurs and the accompanying disturbances are observed.

9. Have you sought first aid.

We talk to parents about information about child development and the history of stuttering. First of all, are the parents healthy: no relatives in the family, suffering from alcoholism, no venereal diseases?

Then it is determined about the child's speech development: During the conversation with parents, information about the child's speech development: initial sounds, loud voices, maundering, first words, when the phrase appeared, how to use the pace of speech, others had special features during communication (stuttering or not stuttering, fast talking, not talking to the child's parents or relatives). Great attention is paid to the upbringing of the child in the family: the attitude of adults to him (pampering or excessive risk, corporal punishment, intimidation); assisting the child in the correct formation of speech (whether there is a violation of the norm in the teaching of complex texts, or, conversely, the complete lack of control over his correct pronunciation of sounds, grammatically correct speech, etc.). When did the stuttering appear, did the first signs of it appear? What does it look like? What are the reasons for this? What other speech defects were observed during this period: in the pronunciation of sounds, its vocabulary and the grammatical structure of sentences, sound, breathing, tempo of speech. Parents paid attention to how the child developed stuttering, what features appeared: whether there was an involuntary movement disorder (twitching, knocking hands, feet, dizziness, etc.) or speech defects (excessive words, sounds, a distinct sound when breathing, and say the words, and so on.)? How does the child react to his or her speech impediment (pays attention, doesn't pay attention, doesn't matter, worries, is embarrassed, hides, is afraid to speak, etc.)? Did the parents ask for help: where, when, what recommendations did they give, what were the results? The child's initiative, movements, imitation, slurred and fast speech, play, production activities, personal characteristics of the stutterer are checked. Once information about the child, including the history of the onset and passage of stuttering, is identified, nonverbal processes that directly affect the stutterer's speech and his or her speech activity are examined. Speech testing requires us to know the speech capabilities of the child, the stored aspect of the speech, and the choice of speech exercises aimed at that. Pictures, poems, fairy tale booklets are used to check children's speech, various toys (cars, dolls, animals, building materials, cubes, dominoes, etc.) are selected. Specific tasks in speech testing include identifying:

- The place and forms of the involvement of the members of the speech;

- the rate of their occurrence and the speech capabilities of the stutterer;

- disorders associated with speech disorders;

- movement disorders accompanied by speech disorders;

- the attitude of the stutterer to his speech defect, the presence of mental characteristics;

Areas of occurrence of contractions (breathing, vocal, articulatory, mixed) and their forms (clonic, tonic, mixed) are determined by sight and hearing. Depending on the results obtained, separate speech exercises aimed at this are selected. Pictures, poems, storybooks are used to check children's speech, various toys (cars, dolls, animals, building materials, cubes, dominoes, etc.) are selected.

During 2017-2019, 800 children with speech defects applied to the LOGOS Center for Educational Correction, of which 385 children underwent speech therapy and 385 children with various types of speech 
defects participated in speech therapy classes. Of these, the number of stuttering children was $135 /(35 \%)$. Of the 135 children who stumbled, 87 children underwent speech therapy. In summary, during the speech therapy examination and during the speech therapy session, we used neuropsychological and binaural alpha-rhythm, Denas therapy, acupressure, and osteopathic correction technologies with speech therapy methods.

\section{DISCUSSION}

Thus, after answering the above questions, it is possible to discuss the causes and some symptoms of stuttering. Such an appearance often results in stuttering as a result of improper use of pedagogical techniques in the upbringing of the child: pampering, memorization of complex works, failure to assist in speech development, adults have a dangerous relationship with the child. The change in the environment in a child's environment, the right attitude to stuttering speech of close people or peers - this is definitely a way to eliminate stigma and warn. Gathering accurate information about the main course of stuttering will help the child to choose the main types of medical and pedagogical effects. For example, when it comes to motor disorders, of course we stop at movement exercises, we can even take therapeutic exercise cycle exercises. When it comes with speech disorders, of course, we get additional exercises, using special methods to teach the child to correct sound deficiencies, vocabulary deficiencies, grammatically correct formation of words. Once information about the child, the history of the onset and passage of stuttering, has been identified, technologies are used that directly affect the stutterer's speech and his or her speech activity. Specific tasks in speech testing include identifying:

- The place and forms of the involvement of the members of the speech;

- the rate of their occurrence and the speech capabilities of the stutterer;

- disorders associated with speech disorders;

- movement disorders accompanied by speech disorders;

- the attitude of the stutterer to his speech defect, the presence of mental characteristics;

Areas of occurrence of contractions (breathing, vocal, articulatory, mixed) and their forms (clonic, tonic, mixed) are determined by sight and hearing. Depending on the results, on the basis of a special medicalpedagogical-psychological approach, speech therapy classes were conducted 3 times a week.

\section{CONCLUSION}

It should be noted that the speech therapy examination of children with stuttering speech defects and the focus on a complex correction-speech therapy effect in the correct development of various aspects of speech gave a good effective, rational, result. Based on the results of this study, the methods of foreign and national scientists (Akimnko VM, Volkova GA, Muminova L.R., Selivyorstov V. I.) and the use of new technologies in speech therapy-correctional-developmental work with children results obtained: high (i.e., fluent, at a moderate rate, without seizures, good speech) $-54 \%$, medium (i.e., fluent, at a moderate rate, without seizures, with good speech, but unfamiliar, in difficult situations, stuttering may occur) - $42 \%$, low level (not eliminated by the end of stuttering in the child) $-4 \%$. formed.

\section{REFERENCES}

[1] Chapter 3 of the Decree of the President of the Republic of Uzbekistan dated December 1, 2017 No. PF-5270 "On measures to radically improve the system of state support of persons with disabilities" bandi.

[2] Decree of the President of the Republic of Uzbekistan No. PF 4947 of February 7, 2017 "On the Action Strategy for the five priority areas of development of the Republic of Uzbekistan for 20172021."

[3] Law of the Republic of Uzbekistan No. ZRU-139 of January 7, 2008 “On Guarantees of the Rights of the Child";

[4] Decree of the President of the Republic of Uzbekistan No. PF-5538 of September 5, 2018 "On additional measures to improve the management system of public education."

[5] Akimenko V.M. Correction of stuttering in children and adults. Rostov-on-Don "Phoenix" 2017, $142 \mathrm{p}$

[6] Belyakova L.I. Speech breathing technique in preschoolers with speech disorders. - M., 2004

[7] Volkova G.A. Play activities in eliminating stuttering in children. -.M., 1983

[8] Jukova N.S., Mastyukova Ye.M., Filichesva T.B., Logopediya. "Fundamentals of theory and practice" M., 2018

[9] State Requirements for the Development of Preschool Children. Compilers: S.S.Mirdjalilova, M.Sh.Rasuleva, D.Sharipova and others. - Tashkent, 2010.

[10] Muminova L.R., Nurkeldieva D.A., Taxirova Sh.R First Aid Methodical manual T. 1918 
[11] Muminova L.R. Speech therapy. Tashkent 2018.Muminova L.R. «Nutqida og ir nuksoni bulgan bolalar bilan ta'lim tarbiya va korreksion ish tizimi». Tashkent 2011.

[12] Selivyorstov V.I. Речевые игры с детьми.- M., 1994

[13] Irgashevich, D. A. (2020). Development of national network (tas-ix). ACADEMICIA: An International Multidisciplinary Research Journal, 10(5), 144-151. Article http://dx.doi.org/10.5958/2249-7137.2020.00254.2 